\title{
A NOVEL APPROACH FOR THE EARLY DETECTION OF PARKINSON'S DISEASE USING EEG SIGNAL
}

\author{
Naveenraj Kamalakannan
}

Undergraduate Student, Department of Communication Engineering, School of Electronics Engineering, Vellore Institute of Technology, Vellore, Tamil Nadu, India

\section{Shiva Prasaath Sudha Balamurugan}

Undergraduate Student, Department of Communication Engineering, School of Electronics Engineering, Vellore Institute of Technology, Vellore, Tamil Nadu, India

\section{Dr. Kalaivani Shanmugam}

Associate Professor, Department of Communication Engineering, School of Electronics Engineering, Vellore Institute of Technology, Vellore, Tamil Nadu, India

\begin{abstract}
Electroencephalography is a test that applies electrophysiological monitoring methods to detect and evaluate the electrical activity of the brain. A Neuronal Dysfunction / Neuronal Death results in behavioral aberration which directly leads to abnormality in the electrical activities of the brain. The objective of this paper is to extract features and analyze the EEG Signal to detect Parkinson's disease at an early stage. This study takes into account several markers subjected to the early detection of PD that includes Welch's power spectral density, Hjorth parameters, Hurst Exponent, Information factors, and other parameters. These computed features quantify the coordination complexity of a windowed EEG data with respect to a person and are modeled to an Artificial Neural Network based classifier with ReLU and Sigmoidal Activation Function to build a classifier with $93.3 \%$ train and $88.17 \%$ test accuracy.
\end{abstract}

Key words: Data Analysis, Disease Detection, Neural Networks, Parkinson's Disease, Signal Processing

Cite this Article: Naveenraj Kamalakannan, Shiva Prasaath Sudha Balamurugan, Kalaivani Shanmugam, A Novel Approach for the Early Detection of Parkinson's Disease using EEG Signal, International Journal of Electrical Engineering and Technology (IJEET), 12(5), 2021, pp. 80-95.

https://iaeme.com/Home/issue/IJEET?Volume $=12 \&$ Issue $=5$ 


\section{INTRODUCTION}

The Neuronal Death, predominantly Substantia Nigra in the Basal Ganglia region of the brain results in Parkinson's Disease (PD). This medical condition is chronic and if diagnosed lately can be life-threatening too. Resting Tremors, Olfactory Loss, Speech Disorders, Cognitive Abnormalities, Sleep Disorders, etc., are early symptoms linked to PD. Other Symptoms include Muscle Rigidity, Constipation, Anxiety, Fatigue, etc. The patients experience NonMotor Symptoms (Depression, Stress, Sleeplessness, etc.) at a very early stage followed by Motor Symptoms (Tremors, Difficult to Move, Speech Impairment, etc.) The two most positive treatments are Deep Brain Stimulation Surgeries and administering L - DOPA drugs. The disease complications are fatal than the disease itself.

According to researches, the reduced levels of dopamine are linked with PD. Dopamine is a major hormone that transmits electrical impulses to control body movements. As the dopamine levels decrease, the coordination between the brain and body tends to become more complex. The actual cause of the disease is still not known. A research carried out by Dr. Friederich Lewy in 1912 showed that there were abnormal aggregations of a protein called "Lewy Bodies" that develop inside Nerve Cells affected by Parkinson's Disease. Currently, scientists are analyzing these protein aggregations to identify the cause of PD.

Accurate and Early Detection of Parkinson's Disease plays a crucial role in slowing down the progress or completely curing it with proper medication. The abnormal electrical activity of the brain can be analyzed to give useful information. Electroencephalography (EEG) is a test that evaluates your brain activity by taking electrical impulse transmissions into account. EEG is a common test, predominantly prescribed for neurodegenerative and neuroinflammation clinical conditions.

The EEG brainwaves are channelized as a linear combination of independent components. Detection and extraction of useful features from this complex brainwave is a challenging task. To add on, the real-time unprocessed EEG signal can have many artifacts that include but not limited to Eye Blinking, Hand Movements, other external factors, etc. Fast Fourier Transform, Welch's Method for Power Spectral Density analysis, and other signal processing techniques are used to extract functional features.

Quantifying the complexity factors as a whole is more significant. This paper analyses the variation of Sample entropy to compute the complexity of coordination of the brain and the body. These computed features play an important role in early diagnoses of PD. This study uses the Independent Component Analysis technique to remove the eye blinking and other artifacts. ICA is a Blind Source Separation technique that extracts statistically independent sources from a set of recorded signals.

This data-driven approach to EEG Signal Processing for Parkinson's Disease has paved way for the extraction of various biomarkers for analysis. In this study, Artificial Neural Network with ReLU and a Sigmoidal activation function is implemented. Researches claim to achieve promising and high accuracy for PD classifier using Gait Analysis [1, 2], and various other techniques. Resting-State EEG microstate maps and analyzes the brain dynamics of Dementia Patients [3] to numerate parameters such as Mean Duration, Frequency of Occurrence and Global explained Variance to classify the PD.

This paper is aimed to extract effective features of EEG signals through proved Mathematical \& ML Algorithms and feed them to an Artificial Neural Network model-based classifier to distinguish PD positive patients with high efficiency and accuracy at the cost of historical data only. 


\section{BACKGROUND}

Identification and treatment of Parkinson's Disease is still a big challenge in the medical industry. The inadequacy of objective and quantifiable biomarkers for diagnosing and monitoring symptoms results in large direct and indirect healthcare costs. A drop in the dopamine-producing neurons causes a variety of motor and non-motor symptoms, but there is presently no conclusive test for PD that can be used by non-specialist physicians, especially in the initial stages of the illness when symptoms are mild and vaguely understood.

Gait analysis is one of the widely used and efficient techniques to detect Parkinson's Disease at an early stage. Few studies have concluded that the positive cases had aberrant Gait patterns in comparison with the healthy controls. Visual assessments of motor activities and speech among other tests, are used in current diagnostic and severity assessment methods and can differ amongst clinicians depending on their competence and visual observation of gait activities. The demerit of Gait analysis is its recording. It can be affected by external factors, which may result in abnormal results.

Veeraragavan et al. (2020) [4] worked on extracting the gait features by using Vertical Ground Reaction Force. An Artificial Neural Network was modeled to diagnose PD using the computed features. The research claims $97.4 \%$ accuracy on disease detection.

Freezing of Gait as a significant feature to detect Parkinson's Disease was proposed by Ashour et al. (2020) [5], which claimed that the desire to walk, freezing of gait (FOG) is characterized as a momentary, sporadic cessation, or substantial decrease in movement of the feet. This research work claims to have achieved $83.38 \%$ accuracy using a Long Short Term Memory (LSTM).

Adams et al. (2017) [6] claims to diagnose Parkinson's Disease using keystrokes of 103 subjects. Various keystroke characteristics were analyzed by an ensemble of machine learning classification models, to diagnose the disease. This research claims to successfully discriminate between early-PD subjects and controls with $96 \%$ sensitivity and $97 \%$ specificity. The goal of this study was to discover the features of finger movement that are influenced by Parkinson's disease (PD) and to use machine learning (ML) to reliably assess the illness status of the study participants. Accurate distinguishing of involuntary and voluntary finger movements is a challenge and can produce unreliable results.

Through these background researches and various exploratory data analyses, in this research work, we will compute multiple features with respect to the characteristics of the EEG data signal. This model will be able to extract the anomalies of this time-series data and use them as a weighted feature for further computations.

\section{METHODOLOGY}

In this research, we have used the BIDS validated OpenNeuro's EEG dataset, which was collected and published by UC San Diego [7]. The resting-state EEG data were collected from 31 patients of which 16 of them are healthy controls and the rest 15 are affected. The data runs for an average of 150 secs and consists of 40 channels. 
A Novel Approach for the Early Detection of Parkinson's Disease using EEG Signal

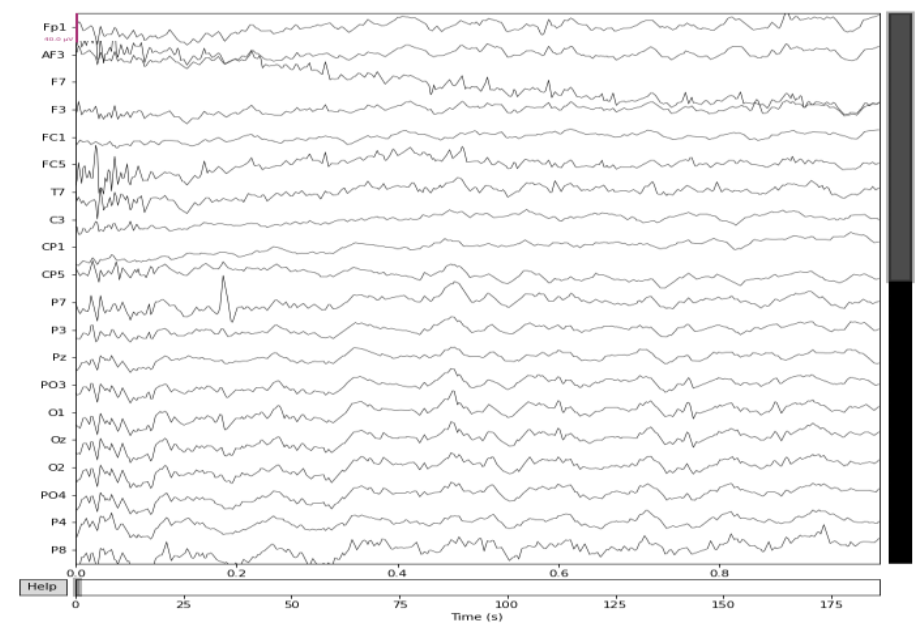

Figure 1 Plot of a collection of EEG channels for a duration of 1 second. Figure 2. Block diagram that represents the flow of process

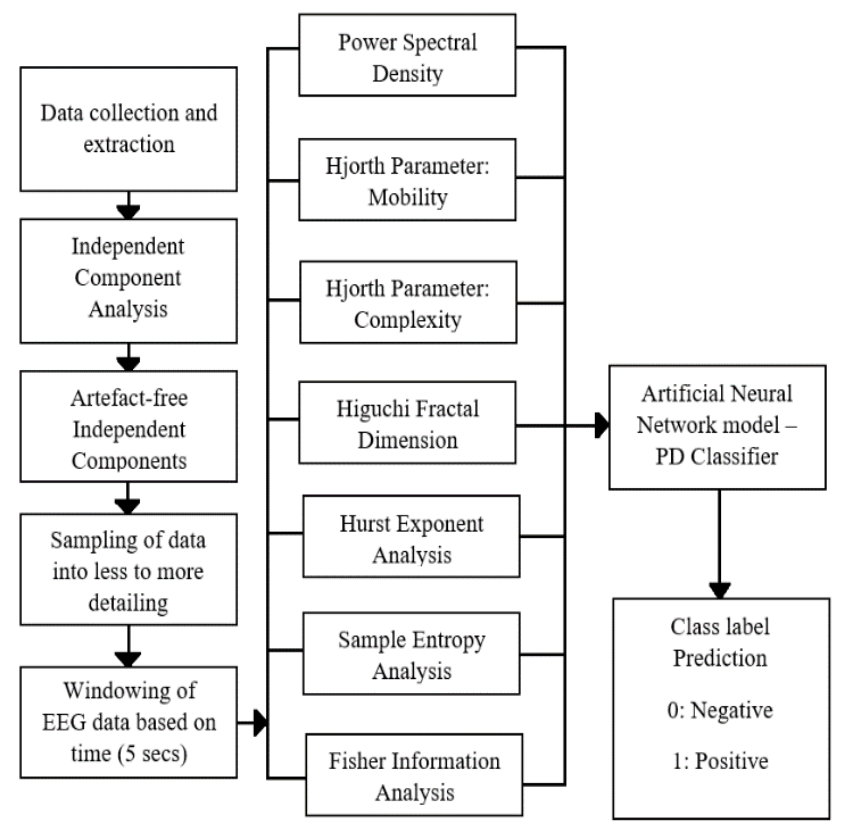

Figure 2 Block diagram that represents the flow of process

This block diagram represents the flow of this research work that includes removing artifacts components, windowing of data, computing of various features, etc. The ANN classifies the subjects into PD positive and healthy controls.

\subsection{Independent Component Analysis}

The dataset consists of 40 channels and is sampled at $512 \mathrm{~Hz}$. The Frontal (F), Temporal (T), Central (C), Parietal (P), and Occipital (O) electrodes follow the internationally recognized EEG Montage.

The first step in EEG signal processing is to eliminate the artifacts so that the outcomes are not affected by high-frequency components. This paper uses the Independent Component Analysis [8], which is a Blind Source Separation (BSS) technique to decompose a signal $s(t)$ into its linearly independent components. This technique computes the unmixing matrix $\mathrm{W}$, where $\bar{S}$ is the $\mathrm{n}-$ unknown independent components and $\mathrm{x}$ is the recorded signals. 


$$
\bar{S}=W \cdot x
$$

First, we separate out the linearly combined data channels into ' $n$ ' independent components using FastICA Algorithm. The next step is to nullify the artifact-rich components and transform the data back into its 40-channel original form.

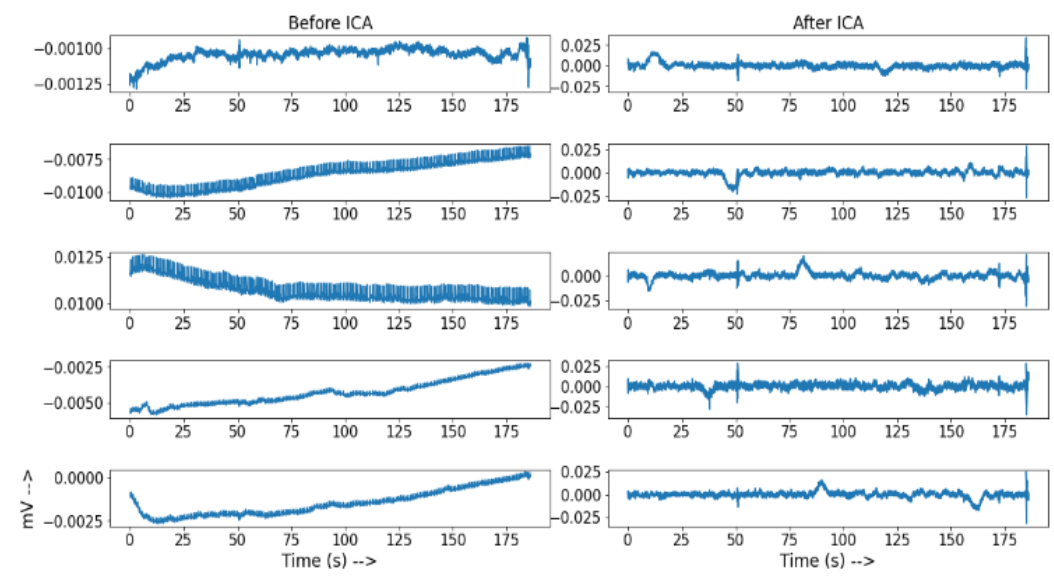

Figure 3 This plot shows the "Before ICA" and "After ICA" representation of a set of 5 channels.

The artifact removal is based on the presupposition that the propagation delays in the recorded time series from the sources of the electrodes are negligible, spatial mixtures and the accumulation of potential collected from different parts are linear. In the "After ICA" section of Figure 3, high-frequency peaks are detected when $t=50$ seconds. A t-sec window runs across the entire channel and a window $\mathrm{w}$ is nullified if it contains any EOG component. This attenuates the effect of EOG components on the succeeding feature computations.

\subsection{Welch's Power Spectral Density Estimate}

EEG Signals are broadly classified into Delta $(<3.5 \mathrm{~Hz})$, Theta $(4-7 \mathrm{~Hz})$, Alpha $(8-13 \mathrm{~Hz})$, and Beta $(14-30 \mathrm{~Hz})$ waves, characterizing the states of vigilance as deep sleep, sleeping, relaxing, and alertness respectively.

In this research, PSD has been computed using the Welch's method [9] by dividing the signal into ' $\mathrm{n}$ ' overlapping segments, estimating a periodogram $\mathrm{P}_{\mathrm{k}}=P_{x_{m}}\left(w_{k}\right)$ by computing the FFT for each window,

$$
P_{x_{m}}\left(w_{k}\right)=\frac{1}{M}\left|F F T_{N, k}\left(x_{m}\right)\right|^{2}=\frac{1}{M}\left|\sum_{n=0}^{N-1} x_{m}(n) e^{\frac{-j 2 \pi n k}{N}}\right|^{2}
$$

where $x_{m}$ is the $m^{\text {th }}$ window of the signal $x$ and the number of available frames is denoted by $\mathrm{K}$, and finally computing

$$
\hat{S}_{x}^{W}\left(w_{k}\right)=\frac{1}{K} \sum_{m=0}^{K-1} P_{x_{m}}\left(w_{k}\right)
$$

the average of the periodogram $\mathrm{P}_{\mathrm{k}}$, where $\hat{S}_{x}^{W}\left(w_{k}\right)$ is the Welch's estimate of the power spectral density. 
A Novel Approach for the Early Detection of Parkinson's Disease using EEG Signal

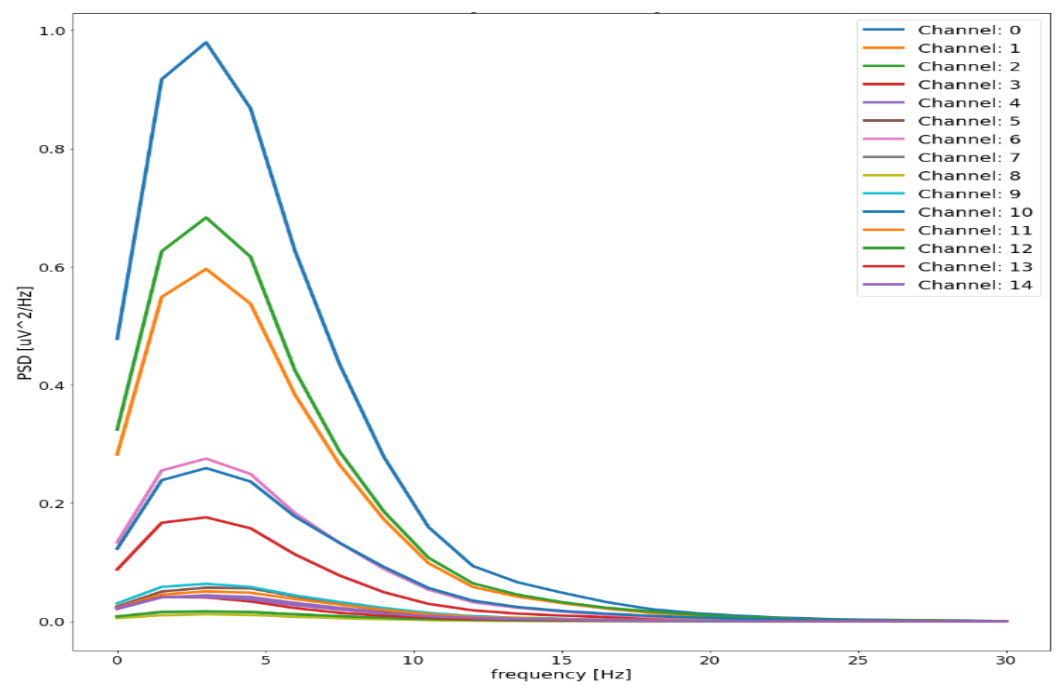

Figure 4(a) The plot shows the Welch's estimate of PSD for the first 15 channels/components of a healthy control

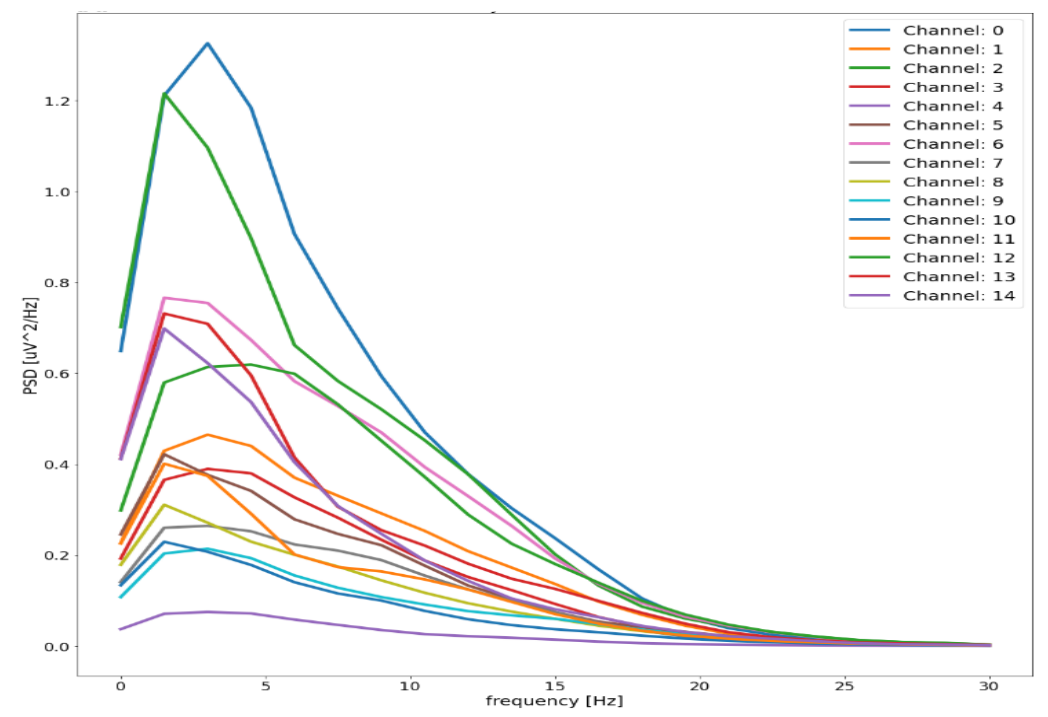

Figure 4(b) The plot shows the Welch's estimate of PSD for the first 15 channels/components of a PD positive subject

Figure 4(a) and 4(b) represents the computed Power Spectral Density which will be used as features for the Machine Learning model-based classifier. They were sampled at $60 \mathrm{~Hz}$ and windowed into 40 segments to decrease the detailing.

In Figure 4(a), the most active region lies between the frequencies, $0 \mathrm{~Hz}$ and $10 \mathrm{~Hz}$. This depicts the resting state of a healthy control while in Figure 4(b), we can observe tumultuous signal data, having significant PSD values in the range of frequencies between $10 \mathrm{~Hz}$ and 15 $\mathrm{Hz}$, depicting a state of tremor in PD Positive subjects. 
Naveenraj Kamalakannan, Shiva Prasaath Sudha Balamurugan, Kalaivani Shanmugam

Channel: 1
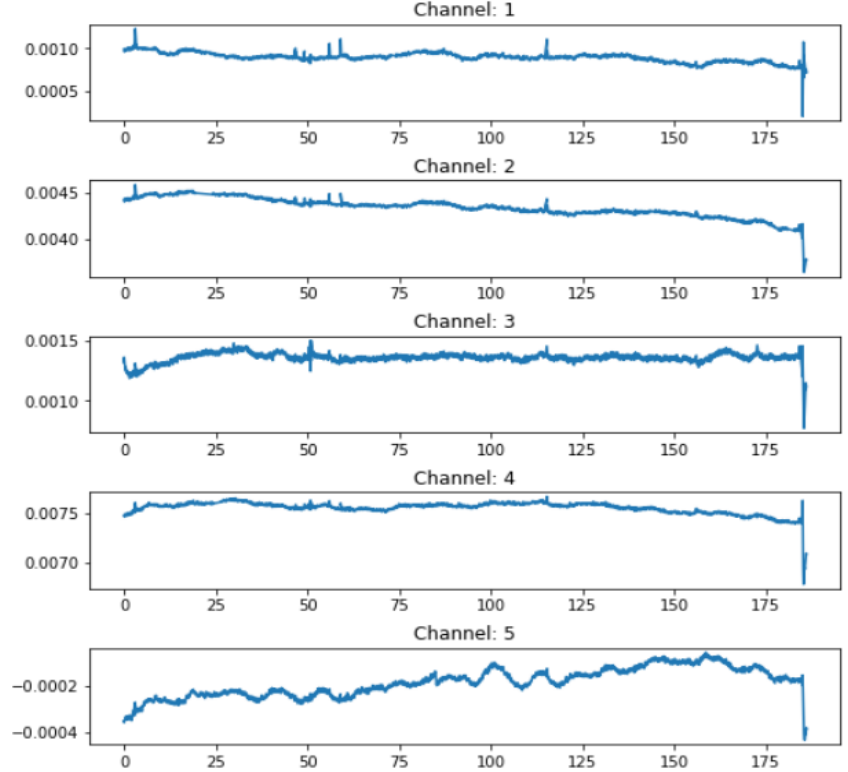

Figure 5 (a)
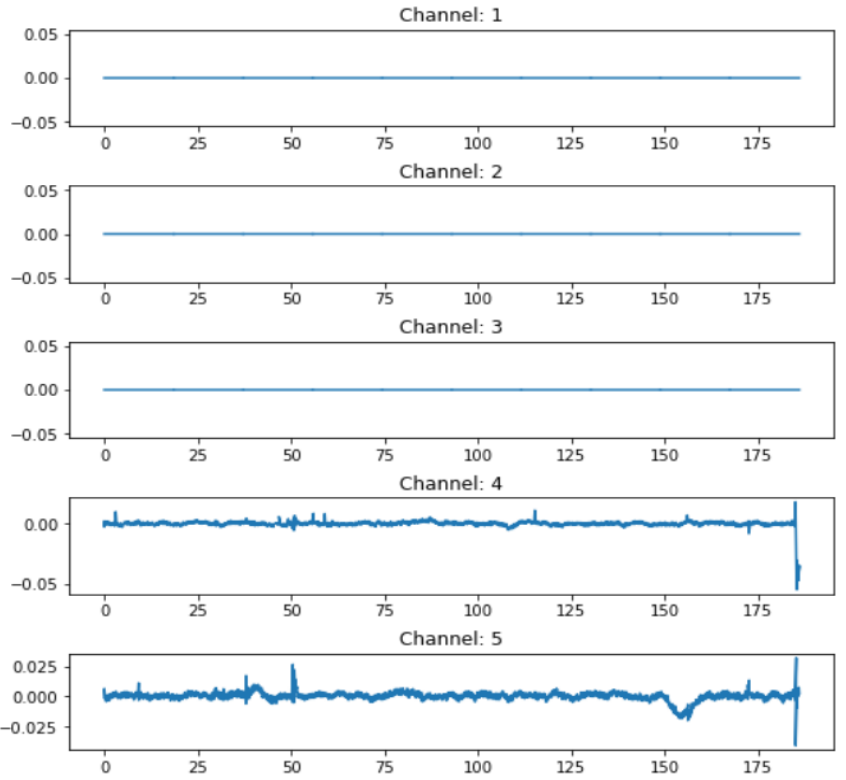

Figure $5(\mathbf{c})$
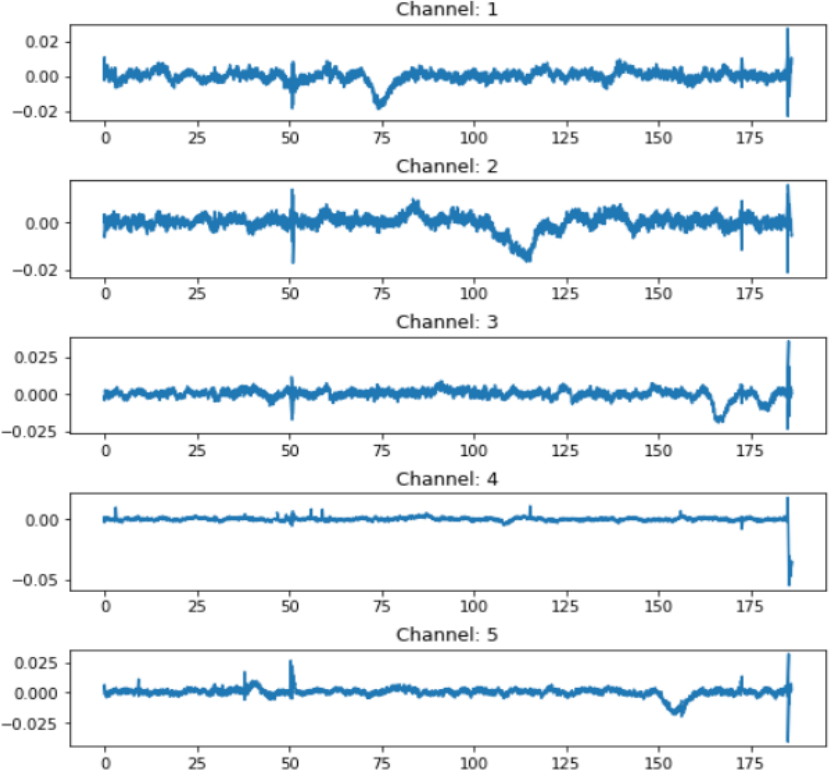

Figure 5 (b)
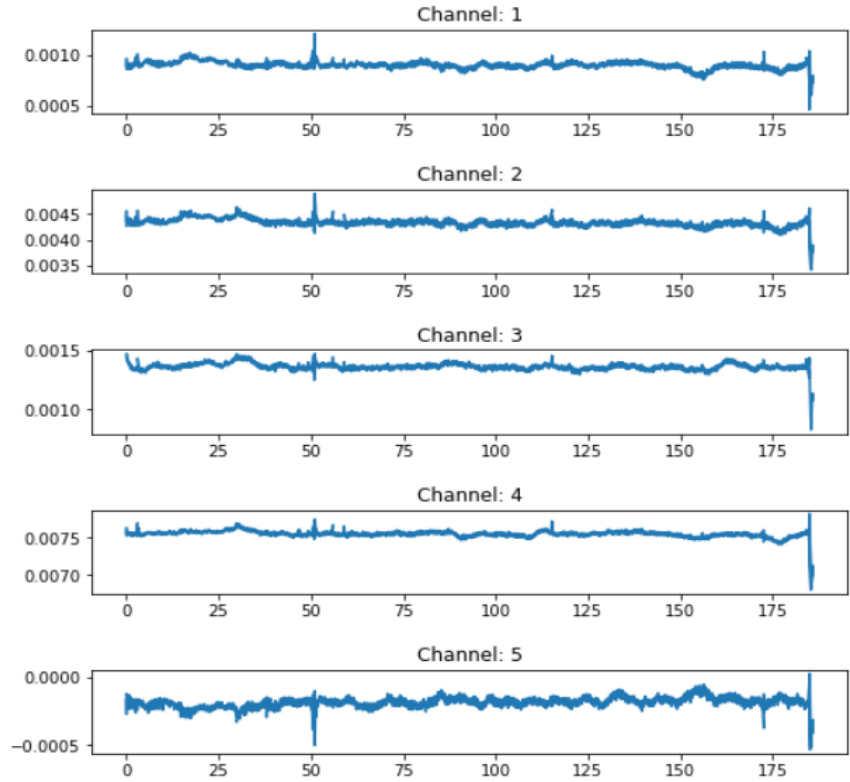

Figure 5 (d)

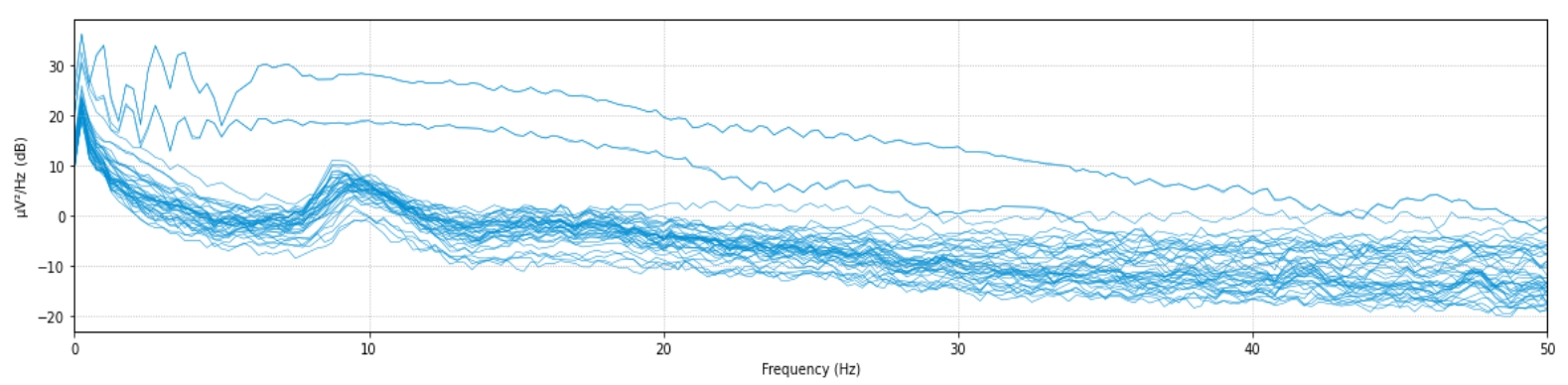

Figure 5 (e)

Figure 5(a), 5(b), 5(c) represents the process of artifact removal using Independent Component Analysis where (a) is the original signal data, (b) is the ICA components, (c) demonstrates the nullifying process and (d) is the ICA inverse transform of the components. Figure 5(e) represents the raw Power Spectral Density of the EEG data with maximum frequency as $50 \mathrm{~Hz}$. For the frequencies lesser than $10 \mathrm{~Hz}$, the PSD values are significant.

This shows that the Delta and Theta waves are predominant. 


\subsection{Sample Entropy}

The data complexity of time-series data is assessed using sample entropy [10]. The data length independence and easier implementation have made it more advantageous than approximate entropy. A higher Sample Entropy value indicates high uncertainty of the system while a lower value indicates self-similar and regular signals.

$$
\operatorname{SampEn}, H(x, m, r)=-\log \frac{C(m+1, r)}{C(m, r)}
$$

where $C(m+1, r)$ is the Chebyshev distance of the embedded vectors of length $m+1$ lesser than the neighborhood's radius $r$, and $C(m, r)$ is the Chebyshev distance of the embedded vectors of length $m$ lesser than $r$.

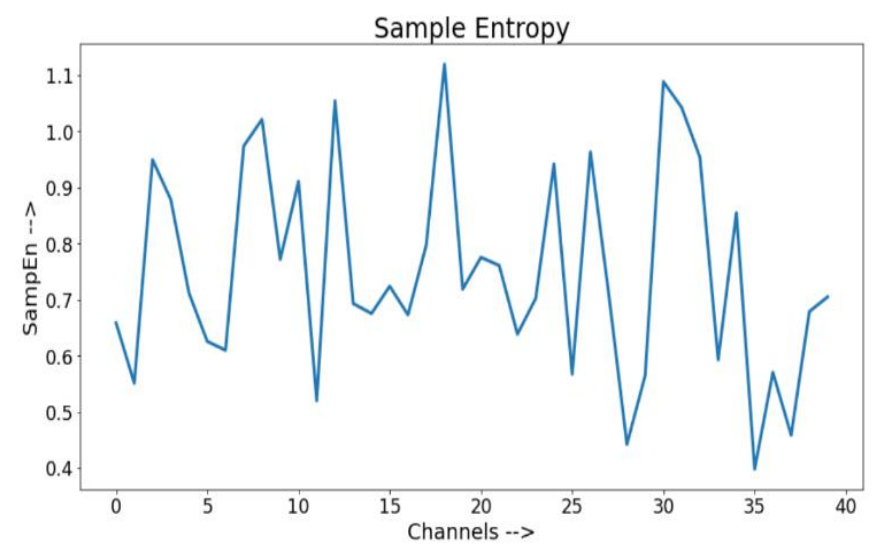

Figure 6 This plot shows the variation of Sample Entropy of all the 40 channel EEG data.

The $16^{\text {th }}, 17^{\text {th }}$ and $18^{\text {th }}$ EEG data channels are highly uncertain while the $28^{\text {th }}$ channel's data is self-similar. These values will be used as a set of features for the classifier model.

\subsection{Hjorth Parameters}

Hjorth parameters are indices of statistical properties for signals in the time domain [11]. These parameters are normalized slope descriptors which are often used in the analysis of EEG signals for feature extraction.

Hjorth Mobility: This denotes the proportion of standard deviation of the power spectrum.

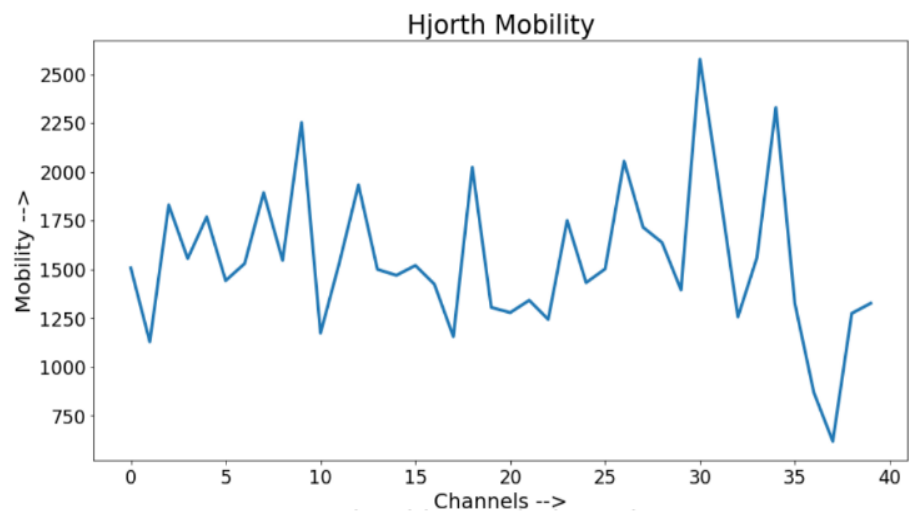

Figure 7 This plot shows the computed Hjorth Mobility across the 40 channels

It is represented as the square root of quotient of variance of the first derivative of the signal $(y(t))$ to that of the variance of signal $(y(t))$. 
Naveenraj Kamalakannan, Shiva Prasaath Sudha Balamurugan, Kalaivani Shanmugam

$$
\text { Mobility }=\sqrt{\frac{\operatorname{var}\left(\frac{d y(t)}{d x}\right)}{\operatorname{var}(y(t))}}
$$

Thus, the result was a feature per channel.

Hjorth Complexity: This indicates the change in frequency and the similarity degree of the signal to a pure sine wave.

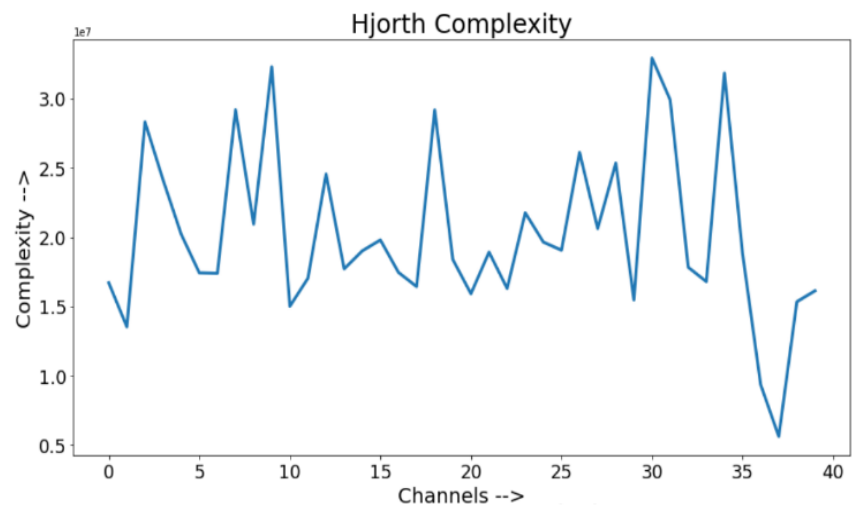

Figure 8 This plot shows the computed Hjorth Complexity across the 40 channels

$$
\text { Complexity }=\sqrt{\frac{\operatorname{Mobility}\left(\frac{d y(t)}{d x}\right)}{\operatorname{Mobility}(y(t))}}
$$

As shown in Figure 8, channel 30 is seen with the highest complexity while channel 37 is the least complex one.

\subsection{Hurst Exponent}

The Hurst exponent is a measure of autocorrelation (persistence and long memory). Hurst exponent [12] describes the long-memory dependence or persistence in the original signal.

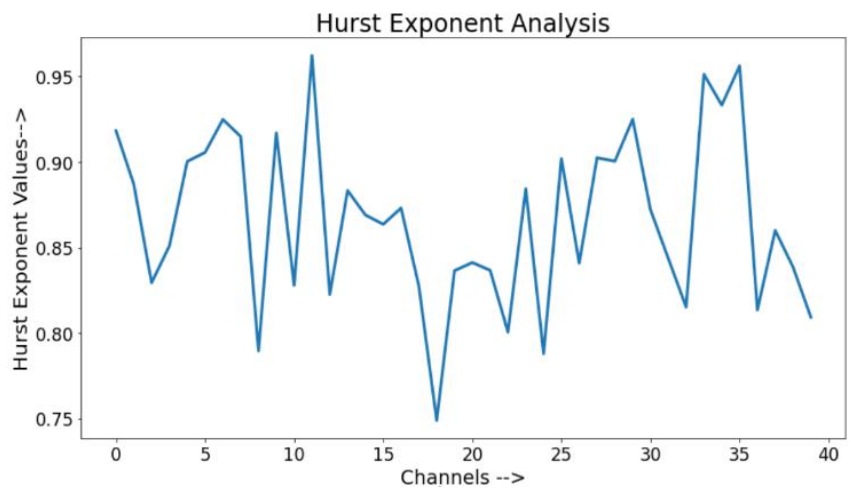

Figure 9 This plot shows the computed Hurst Exponent Values across the 40 channels

From Figure 9, we can see that the Hurst Exponent values are always greater than 0.5 indicating a persistent time series. Channel 9 is the least persistent while channel 33 is the most persistent, compared to other channels. These values will be the most important attribute for the Machine Learning model-based classifier.

\subsection{Higuchi Fractal Dimension}

A fractal dimension is an index for characterizing fractal patterns or sets of the signal by quantifying their complexity as a ratio of the change in detail to the change in scale can be obtained [13]. 


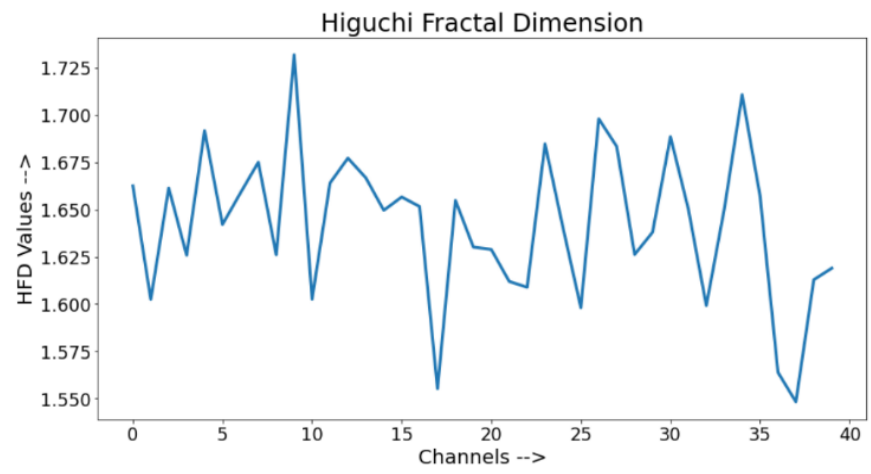

Figure 10 This plot shows the computed Fractal Dimensions values across the 40 channels using Higuchi's algorithm

$\mathrm{L}(\mathrm{k})$ is plotted with $\mathrm{k}^{-1}$ on a $\log -\log$ scale with time interval $\mathrm{k}=1,2 \ldots \mathrm{k}_{\max }$. The slope of the line that exactly fits $\{\ln [L(k)], \ln (1 / k)\}$ in a least square sense is defined as HFD.

$$
L(k)=\frac{1}{k} \sum_{m=1}^{k} L(m, k)
$$

$\mathrm{L}(\mathrm{m}, \mathrm{k})$ is the length of each new time series $X_{k}^{m}$ defined by,

$$
X_{k}^{m}=\left\{x[m], x[m+k], x[m+2 k] . . x\left[m+i n t\left(\frac{N-m}{k}\right) \cdot k\right]\right\}
$$

The initial time value and the length of the original time series is represented as $\mathrm{m}$ and $\mathrm{N}$, respectively. These FD fluctuations across each channel provides insights into the underlying dynamics of the subject. Thus, these values can be used as an important feature for the ML model.

\subsection{Fisher Information}

Fisher information [14] tells us the amount of information about an unknown parameter that can be extracted from the sample. Given the unknown parameter as $\theta$,Fisher Information $I(\theta)$ is given by,

$$
I(\theta)=-\int\left(\frac{\partial^{2}}{\partial \theta^{2}} \log f(x \mid \theta)\right) f(x \mid \theta)
$$

$f(x \mid \theta)$ is the probability density function and the log - likelihood function is given by

$$
l(x \mid \theta)=\log (f(x \mid \theta))
$$

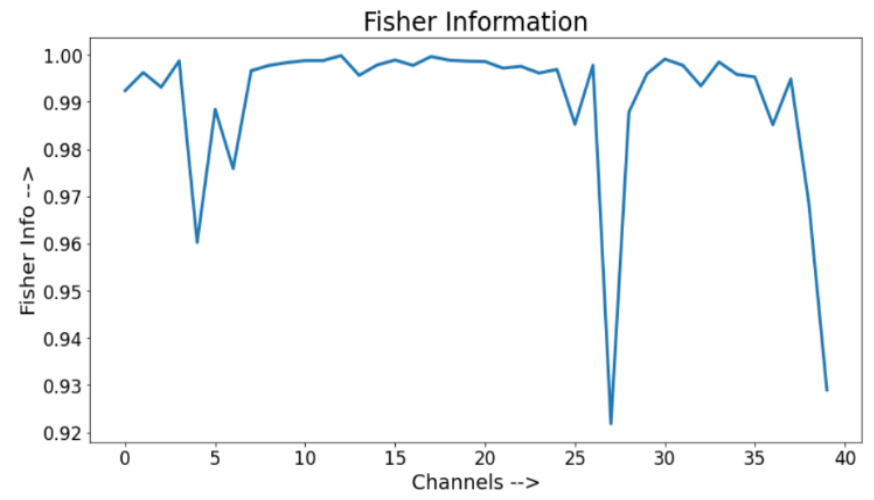

Figure 11 This plot shows the computed Fisher Informationn across the 40 channels 


\section{EXPLORATORY DATA ANALYSIS}

The computed features that include Welch's PSD, Sample entropy values and others are fed to an ANN to classify if the subject is diagnosed with Parkinson's Disease or not. Each patient's data is sliced into a five second window, running across the whole signal data. These windows will have equal weights $\mathrm{w}_{\mathrm{i}}$ and any undesired action that results in an abnormal peak/trough will have minimal influence on the net results obtained.

$$
P S D=\frac{1}{w_{i} * n} \sum_{i=0}^{n-5} w_{i} * P S D(i, i+5)
$$

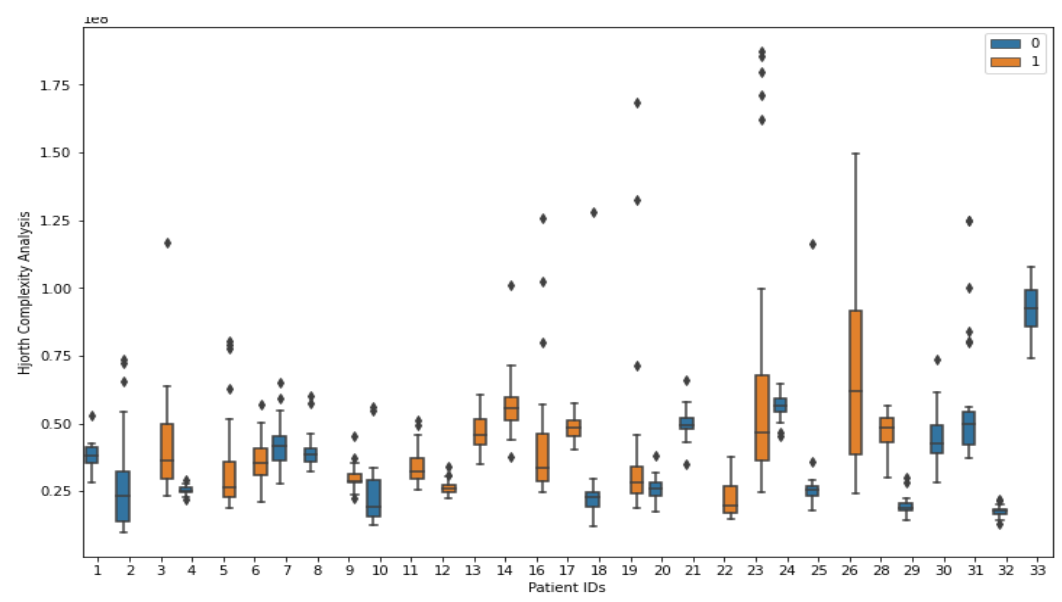

Figure 12 (a): This plot shows the variation of Fisher Information for each of the patient grouped by their class.

Technically, the class imbalance problem is eliminated. Before building the model, performing exploratory data analysis gives an intuition about the data samples. This will be beneficial for choosing the appropriate classifiers.

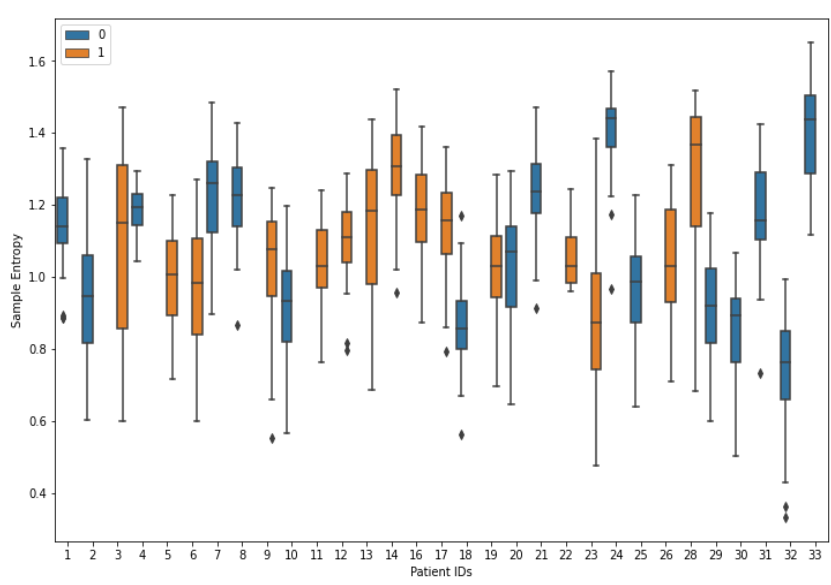

Figure 12 (b): Class 1 shows higher variation of values compared to the healthy controls

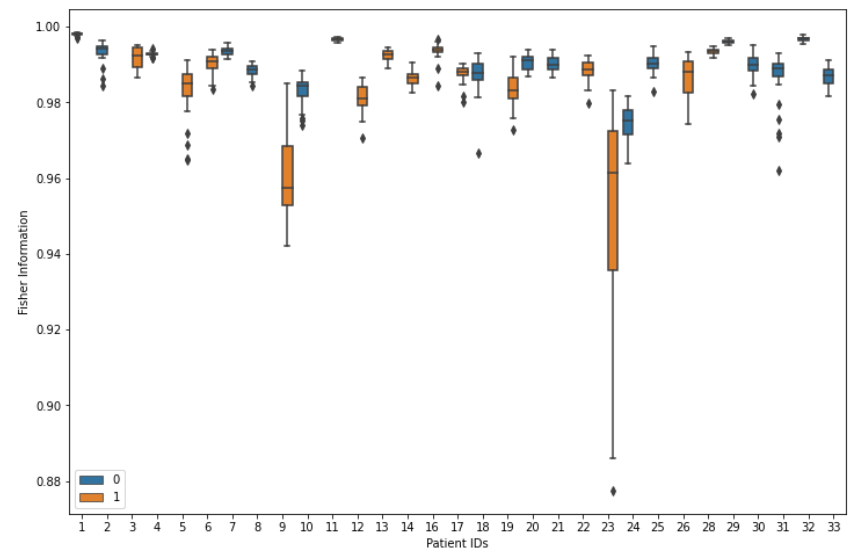

Figure 12 (c): Both the classes 0 and 1 shows the similar variation range for sample entropy 

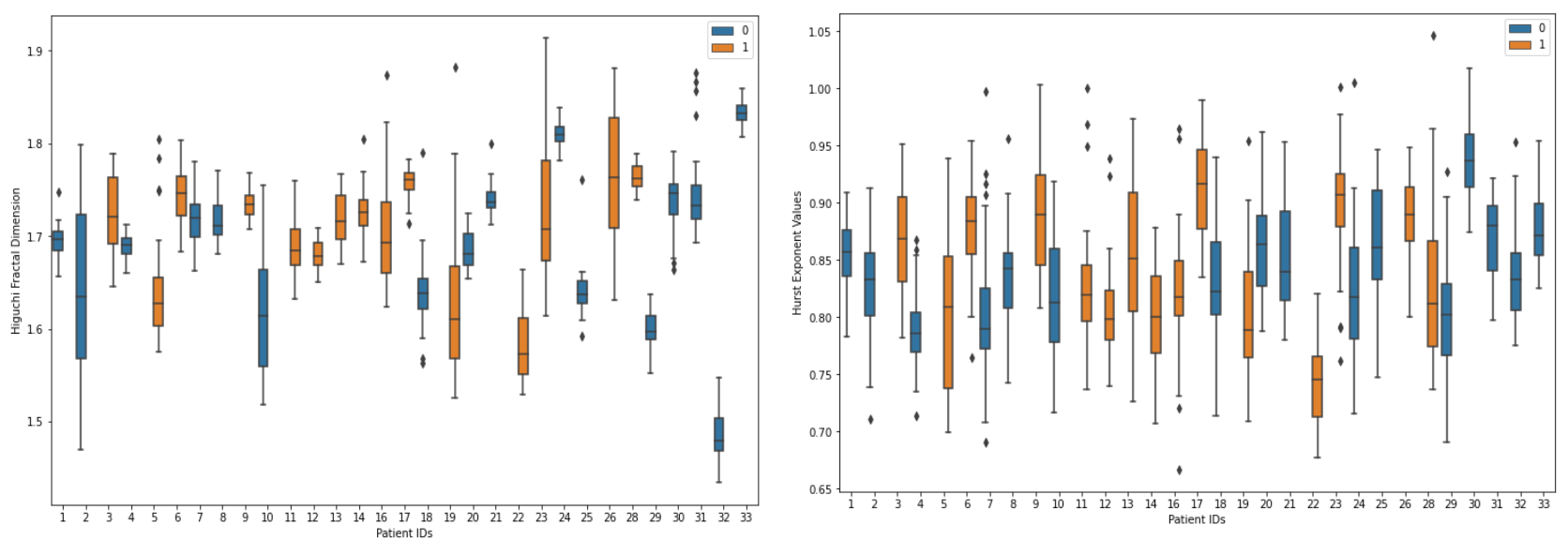

Figure 12 (d): The ranges of variation of both the classes are Figure 12 (e) Both the classes don't show much variation for similar but the value seems to vary. Class 0 occupies majority of the lower half while class 1 occupies the upper half of the plot. This is a significant inference

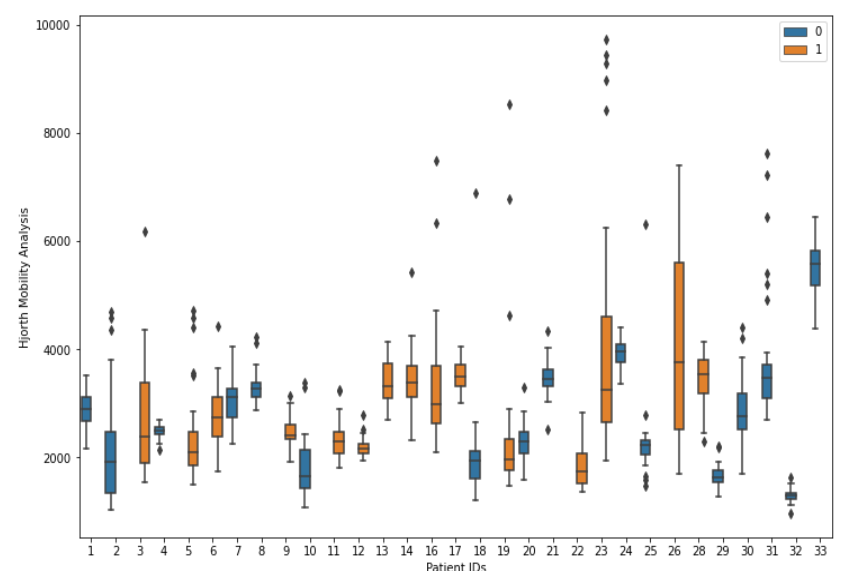

Figure 12 (f): We can observe many outliers in this Hjorth Mobility plot and few class 1 patients have higher range of variations compared to class 0 healthy controls
Power Spectral Density. They are approximately constant

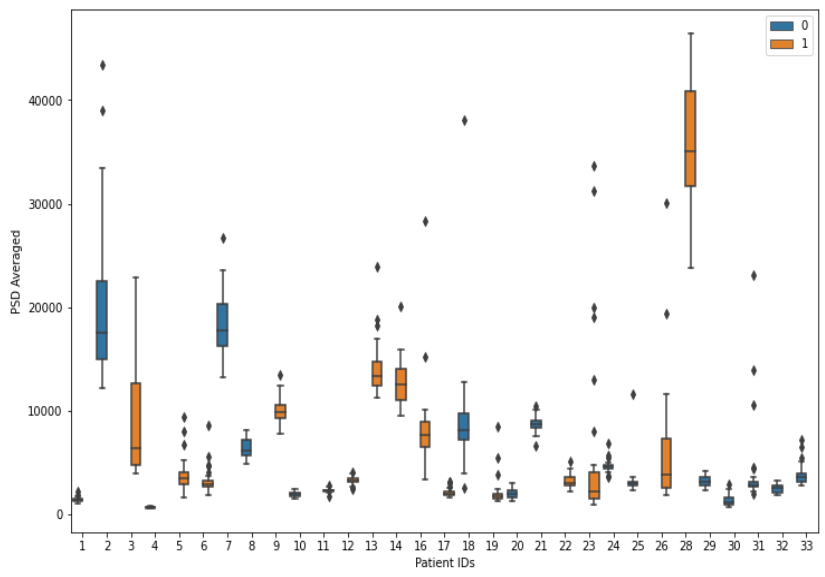

Figure 12 (g): For the hurst exponent box plot, the range of variation is approximately equal for both the classes

The seven feature box-plots in Figure 12 (a), 12 (b), 12 (c), 12 (d), 12 (e), 12 (f) and12 (g) represents the range of variation of Fisher information, Hjorth Complexity, Sample Entropy, HFD, PSD, Hjorth Mobility and Hurst Exponent values with respect to different subjects differentiated using their respective classes. We can infer the value ranges of healthy and affected subjects, and decide upon the scaling factor and choose respective neural network layers. In addition to box plots, line plots and scatter plot can be used to identify the most influential pairs of features, their scaling range, and choose appropriate classifier. In the legend, class ' 0 ' represents healthy control, while class ' 1 ' represents Parkinson's Disease subjects.

\section{BUILDING THE CLASSIFIER}

In this research work, Sequential model of Neural Network [15] is implemented which takes in a stack of layers in an orderly manner. The layers have one input tensor and one output tensor. Our model consists of Flatten layer and Dense layer with ReLU and Sigmoid activation function. 


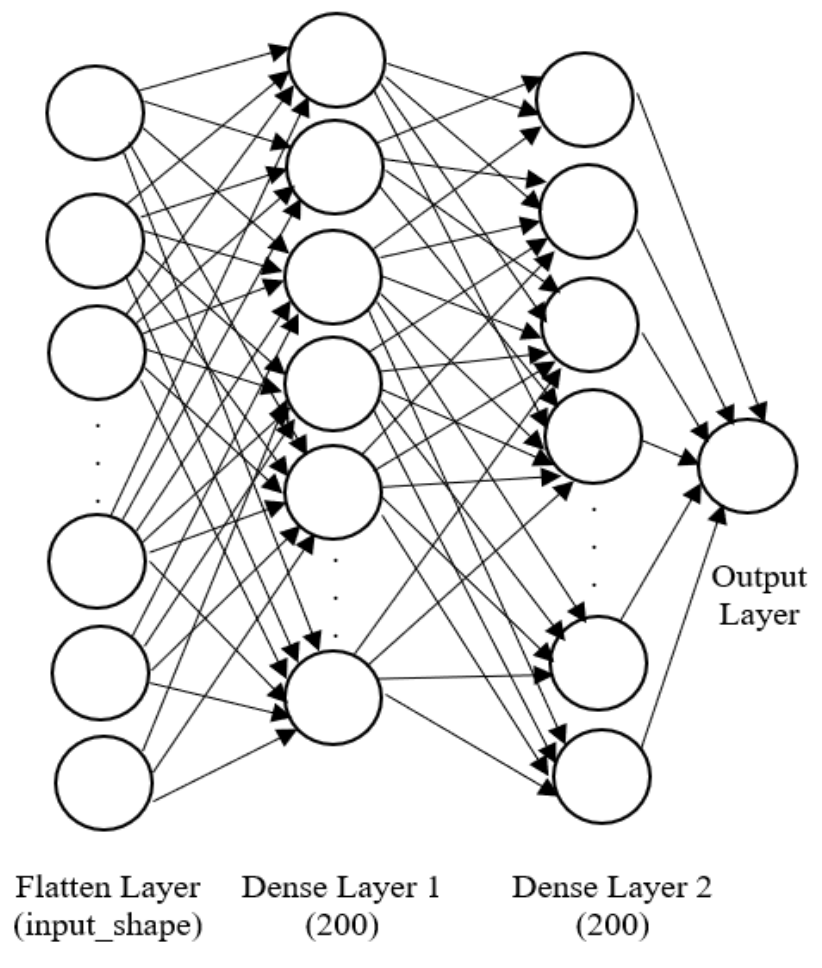

Figure 13. Illustrates the architecture behind the Neural Network Model

\subsection{Dense Layer}

Dense is a neural network layer which is connected deeply and each neuron in this Dense layer [16] receives input from all other layers. The dot products of the inputs are given to an activation function and the output is passed on to the next layer.

\subsection{ReLU Activation Function}

This Rectified Linear Units function will replace all the negative values present with zero (0) in the output matrix and retain all positive values

\subsection{Sigmoid Activation Function}

This Logistic Function transforms the input into a value between 0.0 to 1.0 and is denoted by,

$$
S(x)=\frac{1}{1+e^{-x}}
$$

This function introduces non-linearity into our neural network model.

\section{EVALUATION}

The metrics for ranking this model is Confusion Matrix, and the four important terms to be taken into account are True Positive, False Positive, True Negative, and False Negative.

Table 1 Confusion Matrix for Train Dataset

\begin{tabular}{|c|c|c|c|}
\hline \multirow{4}{*}{$\underset{⿱ 乛}{2}$} & \multirow{2}{*}{$\mathbf{N}=837$} & \multicolumn{2}{|c|}{ PREDICTED } \\
\hline & & Negative & Positive \\
\hline & Negative & 356 & 76 \\
\hline & Positive & 6 & 399 \\
\hline
\end{tabular}


A Novel Approach for the Early Detection of Parkinson's Disease using EEG Signal

Table 2 Important Metrics for Train Dataset

\begin{tabular}{|l|c|}
\hline \multicolumn{1}{|c|}{ Metrics } & Values \\
\hline True Negatives (TN) & 379 \\
\hline True Positives (TP) & 402 \\
\hline False Positives (FP) & 053 \\
\hline False Negatives (FN) & 003 \\
\hline TP Rate (Recall / Sensitivity) & 0.9925 \\
\hline FP Rate & 0.1226 \\
\hline Specificity & 0.8774 \\
\hline Precision & 0.8835 \\
\hline F1 Score & 0.9348 \\
\hline ACCURACY & 0.9330 \\
\hline
\end{tabular}

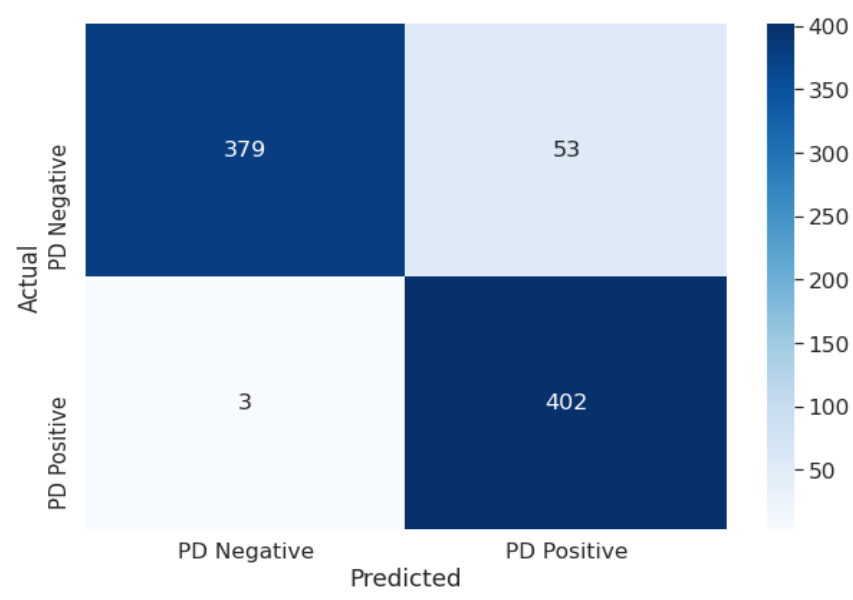

Figure 14. The Confusion matrix of EEG Train Dataset

Table 3 Confusion Matrix for Test Dataset

\begin{tabular}{|c|c|c|c|}
\hline \multirow{4}{*}{ 总 } & \multirow{2}{*}{$\mathbf{N}=93$} & \multicolumn{2}{|c|}{ PREDICTED } \\
\hline & & Negative & Positive \\
\hline & Negative & 37 & 11 \\
\hline & Positive & 0 & 45 \\
\hline
\end{tabular}

Table 4 Important Metrics for Test Dataset

\begin{tabular}{|l|c|}
\hline \multicolumn{1}{|c|}{ Metrics } & Values \\
\hline True Negatives (TN) & 037 \\
\hline True Positives (TP) & 045 \\
\hline False Positives (FP) & 011 \\
\hline False Negatives (FN) & 000 \\
\hline $\begin{array}{l}\text { TP Rate (Recall / } \\
\text { Sensitivity) }\end{array}$ & 1.0000 \\
\hline FP Rate & 0.2291 \\
\hline Specificity & 0.7708 \\
\hline Precision & 0.8035 \\
\hline ACCURACY & 0.8817 \\
\hline
\end{tabular}




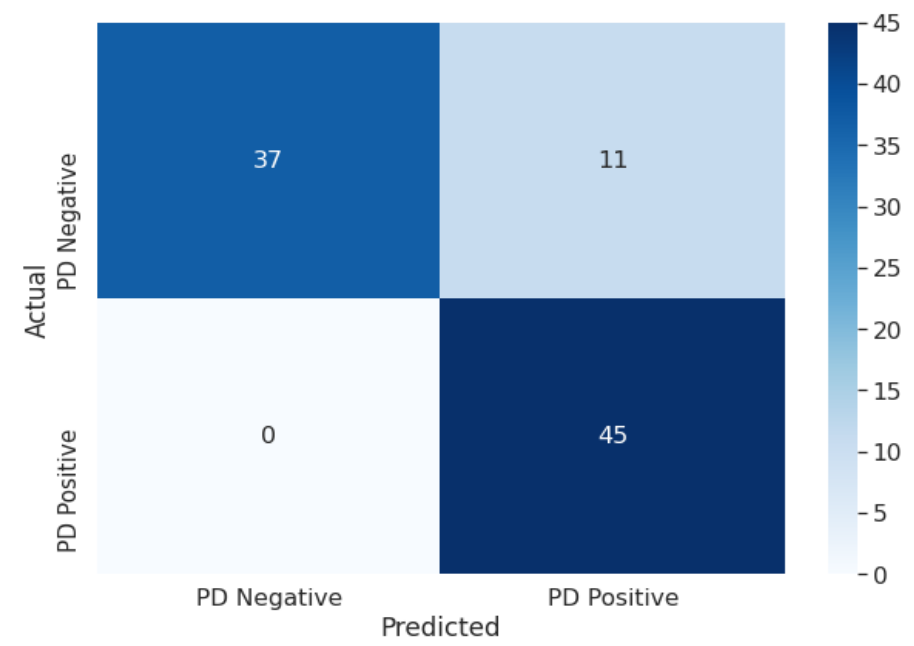

Figure 15 The Confusion matrix of EEG Test Dataset

\section{CONCLUSION AND FUTURE WORK}

In this research work, an Artificial Neural Network model has been built for classifying Parkinson's Disease. This model produces an accuracy of $93.3 \%$ and a test set accuracy of $88.17 \%$. The above analysis suggests that the Parkinson's disease has the potential to be classified at above chance accuracy with this model with the major caveat that the patients in this study are all clinically diagnosed with Parkinson's already and thus the sensitivity of this model applies to patients who have been experiencing symptoms for several years.

The research work has been acknowledged by Mr. Alexander P. Rockhill, University of Oregon, who has no qualms in publishing this paper with the above-mentioned accuracy metrics.

\section{REFERENCES}

[1] Amira S. Ashour, Amira El-Attar, Nilanjan Dey, Hatem Abd El-Kader, Mostafa M. Abd ElNaby, "Long short term memory based patient-dependent model for FOG detection in Parkinson's disease", Pattern Recognition Letters, Volume 131, https://doi.org/10.1016/j.patrec.2019.11.036.

[2] Imanne El Maachi, Guillaume-Alexandre Bilodeau, Wassim Bouachir, "Deep 1D-Convnet for accurate Parkinson disease detection and severity prediction from gait", Expert Systems with Applications, Volume 143, 2020, 113075, ISSN 0957-4174, https://doi.org/10.1016/j.eswa.2019.113075.

[3] Pal, A., Behari, M., Goyal, V. et al. "Study of EEG microstates in Parkinson's disease: a potential biomarker?”. Cogn Neurodyn 15 (2021). https://doi.org/10.1007/s11571-020-096430

[4] W. Wang, J. Lee, F. Harrou and Y. Sun, "Early Detection of Parkinson's Disease Using Deep Learning and Machine Learning," in IEEE Access, vol. 8, pp. 147635-147646, 2020, doi: 10.1109/ACCESS.2020.3016062.

[5] Veeraragavan S, Gopalai AA, Gouwanda D and Ahmad SA (2020) Parkinson's Disease Diagnosis and Severity Assessment Using Ground Reaction Forces and Neural Networks. doi: 10.3389/fphys.2020.587057

[6] Adams WR (2017) High-accuracy detection of early Parkinson's Disease using multiple characteristics of finger movement while typing. PLoS ONE 12(11): e0188226. https://doi.org/10.1371/journal.pone.0188226 
[7] Alexander P. Rockhill and Nicko Jackson and Jobi George and Adam Aron and Nicole C. Swann (2020). "UC San Diego Resting State EEG Data from Patients with Parkinson's Disease". doi: 10.18112/openneuro.ds002778.v1.0.2

[8] Lisha Sun, Ying Liu and P. J. Beadle, "Independent component analysis of EEG signals," Proceedings of 2005 IEEE International Workshop on VLSI Design and Video Technology, DOI: 10.1109/IWVDVT.2005.1504590

[9] P. Welch, "The use of fast Fourier transform for the estimation of power spectra: A method based on time averaging over short, modified periodograms, " in IEEE Transactions on Audio and Electroacoustics, vol. 15, no. 2, pp. 70-73, June 1967, doi: 10.1109/TAU.1967.1161901.

[10] George J. A. Jiang, Shou-Zen Fan, Maysam F. Abbod, Hui-Hsun Huang, Jheng-Yan Lan, FengFang Tsai, Hung-Chi Chang, Yea-Wen Yang, Fu-Lan Chuang, Yi-Fang Chiu, Kuo-Kuang Jen, Jeng-Fu Wu, Jiann-Shing Shieh, "Sample Entropy Analysis of EEG Signals via Artificial Neural Networks to Model Patients' Consciousness Level Based on Anesthesiologists Experience", BioMed Research International, vol. 2015, Article ID 343478, 8 pages, 2015. https://doi.org/10.1155/2015/343478

[11] Vourkas, M. \& Sifis, Micheloyannis \& Papadourakis, Giorgos. (2000). "Use of ANN and Hjorth parameters in mental-task discrimination." Advances in medical signal and information processing, 2000 First international conference on (IEE conf publ no 476). 327 - 332. 10.1049/cp:20000356.

[12] Shujuan Geng, Weidong Zhou, Qi Yuan, Dongmei Cai \& Yanjun Zeng (2011) "EEG non-linear feature extraction using correlation dimension and Hurst exponent," Neurological Research, 10.1179/1743132811Y.0000000041

[13] A. H. Al-nuaimi, E. Jammeh, L. Sun and E. Ifeachor, "Higuchi fractal dimension of the electroencephalogram as a biomarker for early detection of Alzheimer's disease," 2017 39th Annual International Conference of the IEEE Engineering in Medicine and Biology Society (EMBC), 2017, pp. 2320-2324, doi: 10.1109/EMBC.2017.8037320.

[14] Julio Ramírez-Pacheco, Homero Toral-Cruz, Luis Rizo Domínguez, Joaquin Cortez-Gonzalez, "Generalized Wavelet Fisher's Information of $1 /(f)^{\alpha}$ Signals", Advances in Mathematical Physics, vol. 2015, Article ID 210592, 9 pages, 2015. https://doi.org/10.1155/2015/210592

[15] Visakan Kadirkamanathan, Mahesan Niranjan; “A Function Estimation Approach to Sequential Learning with Neural Networks." Neural Comput 1993; 5 (6): 954-975. doi: https://doi.org/10.1162/neco.1993.5.6.954

[16] L. Zhang, D. Borggreve, F. Vanselow and R. Brederlow, "Quantization Considerations of Dense Layers in Convolutional Neural Networks for Resistive Crossbar Implementation, " 2020 9th International Conference on Modern Circuits and Systems Technologies (MOCAST), 2020, pp. 1-6, doi: 10.1109/MOCAST49295.2020.9200280. 